\title{
Gestión del servicio de préstamos interbibliotecarios del Acuerdo de Bibliotecas Universitarias de la provincia de Córdoba (ABUC)
}

\author{
María Luz Chaves \\ Universidad Nacional de Córdoba. Facultad de Filosofia y Humanidades. Escuela de Bibliotecología. Córdoba, Argentina | \\ mlchaves@ffyh.unc.edu.ar / https://orcid.org/oooo-0o01-5073-7244 \\ Sandra Gisela Martín \\ Universidad Nacional de Córdoba. Facultad de Filosofia y Humanidades. Escuela de Bibliotecología. Córdoba, Argentina | \\ sandragmartin@gmail.com / https://orcid.org/oooo-0o03-4045-4915
}

\begin{abstract}
Resumen
El presente trabajo describe la gestión de los préstamos interbibliotecarios (PI) del Acuerdo de Bibliotecas Universitarias de Córdoba. Se plantearon como objetivos para determinar el nivel de uso del servicio, el porcentaje de documentos obtenidos sobre el total de peticiones realizadas, la aplicación de encuestas de satisfacción, el uso de sistemas de promoción/difusión, la tipología de usuarios que utilizan el servicio, la tipología de documentos que solicitan, la forma en que las bibliotecas reciben las peticiones, las formas de envío utilizadas y los costos asociados al servicio. Se identificaron además las fortalezas y debilidades respecto a la logística y a la gestión del servicio, el nivel de satisfacción respecto al software de gestión y el nivel de conocimiento respecto al reglamento de PI. Se llevó a cabo un estudio exploratorio descriptivo con enfoque cuantitativo. El diseño fue transversal enmarcado en un estudio de campo cuyas unidades de análisis fueron los bibliotecarios encargados del servicio de PI y los directores de las bibliotecas miembros de ABUC. Se pudo concluir que la mayor fortaleza del servicio está en el acceso a otras colecciones, lo que permite ampliar el acervo bibliográfico disponible para los usuarios. Las mayores debilidades del servicio de préstamos interbibliotecarios estuvieron relacionadas con los problemas relativos al software Cor-pib y con el envío y recepción de los documentos.
\end{abstract}

\section{Management of the inter-library loan service of the Cordoba University Library Agreement (ABUC)}

\footnotetext{
Abstract

This work describes the management of interlibrary loans (PI) of the Córdoba University Libraries Agreement. The objectives to determine the level of use of the service,

\section{Palabras clave}

Préstamos interbibliotecarios Cooperación bibliotecaria Bibliotecas universitarias Estadísticas Argentina

Keywords ABUC Interlibrary loans Library cooperation University libraries Statistics Argentine 
the percentage of documents obtained over the total number of requests made, the application of satisfaction surveys, the use of promotion / dissemination systems, the typology of users who use the service, the type of documents they request, the way libraries receive requests, the shipping methods used and the costs associated with the service were establised. It was also sought to identify the strengths and weaknesses regarding logistics and service management, the level of satisfaction with management software and the level of knowledge regarding IP regulations. A descriptive exploratory study with a quantitative approach was established. The design was cross-sectional framed in a field study whose units of analysis were the librarians in charge of the IP service and the directors of the ABUC member libraries. It was concluded that the greatest strength of the service is in access to other collections, which allows expanding the bibliographic heritage available to users. The major weaknesses of the interlibrary loan service were related to the problems related to the Cor-pib software and the sending and receiving of documents.

Artículo recibido: 27-03-2020. Aceptado: 23-10-2020

\section{Introducción}

Las bibliotecas universitarias enfrentan el reto de garantizar el acceso al conocimiento y para ello no trabajan solas, sino a través de la cooperación bibliotecaria que se presenta como un pilar fundamental para hacer frente a este desafío. Las relaciones horizontales entre personas e instituciones se ven hoy favorecidas por las tecnologías de la comunicación e información y el trabajo en conjunto produce resultados positivos y enriquece la diversidad, por lo tanto, la cooperación bibliotecaria es esencial si se pretende la ampliación, la mejora, la innovación y la mayor competitividad en la oferta de servicios bibliotecarios.

En el año 2001 los rectores de las universidades de Córdoba firmaron el Acuerdo de Bibliotecas Universitarias de la provincia de Córdoba - ABUC (ABUC, 2018a), constituyéndose así la primera experiencia de cooperación formal entre bibliotecas de instituciones públicas y privadas y en el año 2002, se firmó el Acta de Constitución del Programa de Cooperación. A lo largo de su historia, ha logrado el apoyo claro y firme por parte de las autoridades de las instituciones miembros, el convencimiento de la necesidad de cooperación en materia de información y el sentimiento de compartir beneficios mutuos entre las universidades cordobesas (Martín, 2013).

Actualmente, ABUC nuclea a 46 bibliotecas, reúne más de un millón de documentos y recopila casi 18.000 tesis y trabajos finales. Está conformado por 13 instituciones -12 universidades y 1 institución gubernamental - de la provincia de Córdoba:

» Ministerio de Ciencia y Tecnología del Gobierno de Córdoba (ex Agencia Córdoba Ciencia) - público.

»Universidad de la Defensa Nacional (UNDEF), ex Instituto Universitario Aeronáutico (IUA) -nacional.

» Universidad Católica de Córdoba (UCC) - privada.

"Universidad Blas Pascal (UBP) - privada.

» Universidad Empresarial Siglo 21 (UES 21) -privada.

» Universidad Nacional de Córdoba (UNC) -pública.

"Universidad Nacional de Río Cuarto (UNRC) -pública.

» Universidad Nacional de Villa María (UNVM) -pública. 
» Universidad Tecnológica Nacional:

Facultad Regional Córdoba (UTNFRC) - pública.

Facultad Regional San Francisco (UTNFSFCO) - pública.

Facultad Regional Villa María (UTNFRVM) -pública.

» Universidad Provincial de Córdoba (UPC) - pública.

» Instituto Universitario de Ciencias Biomédicas de Córdoba (IUCBC) - privada.

Los productos y servicios cooperativos consolidados son: los préstamos interbibliotecarios (PI), el boletín electrónico de noticias, los eventos de capacitación gratuitos destinados a directivos y a personal de las bibliotecas miembros, la plataforma digital de gestión de los préstamos interbibliotecarios Cor-pib, el repositorio digital de investigaciones científicas y tecnológicas Cor-ciencia, la plataforma virtual de gestión de materiales duplicados o destinados a canje y/o donación Cor-dupli, el directorio de bibliotecas académicas de Córdoba Cor-dir, el catálogo colectivo de tesis de doctorado, maestrías y trabajos finales Cor-tesis, el catálogo colectivo Cor-cat, la bolsa de trabajo, pasantías y becas Cor-labora y los encuentros anuales Quién es Quién.

El servicio de préstamo interbibliotecario se formalizó en el año 2002 mediante la firma por parte de los rectores del Reglamento correspondiente (ABUC, 2018b). Años más tarde, se desarrolló el software de gestión Cor-pib y el instructivo para su uso (ABUC, 2013). El software dispone de tres módulos: el primero para pedidos de PI (creación de pedidos, consulta de solicitudes recibidas, consultas de pedidos realizados, historial de pedidos y solicitudes anuladas), el segundo para comunicaciones (envío y recepción de mensajes y listado de contactos) y el tercero para informes (estadísticas y exportación de reportes).

\section{Objetivos y metodología}

El objetivo de este trabajo fue describir la gestión del servicio de PI del ABUC desde dos dimensiones. En primer lugar, respecto a la gestión del préstamo interbibliotecario en sí mismo, donde se buscó determinar el tiempo promedio de gestión del servicio, el nivel de uso en los últimos años, establecer el porcentaje de documentos obtenidos sobre el total de peticiones realizadas, identificar el uso de encuestas de satisfacción y/o algún sistema de promoción o difusión del servicio, identificar la tipología de usuarios y de documentos, la forma en que las bibliotecas reciben las peticiones, las formas de envío utilizadas, los costos asociados y los mecanismos para afrontarlos.

En segundo lugar, se indagó a bibliotecarios y directivos para identificar las fortalezas y debilidades respecto a la logística y a la gestión del servicio, para conocer el nivel de satisfacción respecto al software de gestión Cor-pib y para establecer el nivel de conocimiento respecto al reglamento de PI.

Se trató de un estudio exploratorio descriptivo con un enfoque cuantitativo y con un diseño de investigación transversal. La investigación se enmarcó en un estudio de campo cuyas unidades de análisis fueron los bibliotecarios encargados del servicio de PI y los directores de las bibliotecas miembros de ABUC. El universo lo constituyeron la totalidad de las 46 bibliotecas y la población de estudio estuvo formada por una muestra no probabilística por conveniencia. Dado que algunas instituciones poseen varias bibliotecas se optó por seleccionar solo algunas de ellas. Para establecer la muestra se procedió a seleccionar de la Universidad Nacional de Córdoba (UNC), solo aquellas bibliotecas pertenecientes a las 15 facultades más la Biblioteca Mayor que depende del rectorado; de la Universidad Católica de Córdoba (UCC) solo la biblioteca central por ser la biblioteca que centraliza el servicio de PI y de la Universidad Provincial de Córdoba (UPC) la biblioteca del Instituto Cabred por ser, por 
el momento, la única biblioteca que participa del servicio de PI. De esta manera, la muestra quedó conformada por 26 bibliotecas universitarias:

1. UNDEF / Biblioteca.

2. UBP / Biblioteca.

3. UCC / Biblioteca.

4. UES 21 / Biblioteca.

5. UNC / Biblioteca. Facultad de Arquitectura, Urbanismo y Diseño.

6. UNC/ Biblioteca. Facultad de Artes.

7. UNC / Biblioteca Facultad de Ciencias Agropecuarias.

8. UNC / Biblioteca Facultad de Ciencias Médicas.

9. UNC / Biblioteca Facultad de Ciencias Químicas.

10.UNC / Biblioteca Facultad de Ciencias Económicas.

11.UNC / Biblioteca Facultad de Filosofía y Humanidades- Psicología

12.UNC / Biblioteca Facultad de Lenguas.

13.UNC / Biblioteca Facultad de Matemática, Astronomía, Física y Computación.

14.UNC / Biblioteca Facultad de Odontología.

15.UNC / Biblioteca Facultad de Ciencias Exactas, Físicas y Naturales (Centro).

16.UNC / Biblioteca Facultad de Derecho y Ciencias Sociales.

17.UNC / Biblioteca Facultad de Comunicación.

18.UNC / Biblioteca Facultad de Ciencias Sociales.

19.UNC / Biblioteca Mayor.

20.UNRC / Biblioteca.

21.UNVM / Biblioteca.

22.UTN-FRC / Biblioteca.

23.UTN-FRSF / Biblioteca.

24.UTN-FRVM / Biblioteca.

25.UPC / Biblioteca del Instituto Cabred

26.IUCBC / Biblioteca.

La investigación de campo se realizó a partir de las encuestas y el instrumento de recolección de datos se desarrolló mediante un cuestionario semiestructurado diseñado con preguntas abiertas y cerradas que fueron administrados personalmente y/o por correo electrónico. El trabajo de campo comenzó en el mes de agosto de 2018 y finalizó en octubre del mismo año. Se aplicaron los cuestionarios a los 26 encargados de PI y se obtuvieron 25 respuestas. Solo en una biblioteca el encargado no respondió porque no realizan PI, razón por la cual se consideraron las 25 encuestas como el 100\% de las respuestas. Respecto a las encuestas a los 26 directivos se obtuvieron en su totalidad.

\section{Marco teórico}

Martínez de Sousa (1989: 575) define al PI como el "intercambio por parte de sus fondos de dos o más bibliotecas", cuyo propósito es fomentar la cooperación con objeto de hacer accesible al usuario de una biblioteca la colección de otra, para su consulta o investigación. De acuerdo a Magán Wals (1998: 281) "el préstamo interbibliotecario es el medio más eficaz para garantizar el acceso global a la información". Los préstamos implican un contrato bilateral, con derechos y obligaciones recíprocos para el usuario y la biblioteca y se ajustan a las disposiciones de un reglamento que por lo general debería establecer: los requisitos necesarios para la realización del préstamo, los usuarios que pueden solicitar el servicio, la cantidad y el tipo de documentos a prestar, el período de tiempo estipulado y la penalización cuando el préstamo no se devuelve en el tiempo establecido. 


\section{Procedimiento generalizado del PI}

Cuando el usuario busca un documento en el catálogo de la biblioteca (A) y no lo encuentra recurre a consultar catálogos colectivos y/o catálogos de otras bibliotecas, donde puede localizar el material que necesita, en otra biblioteca (B). Realiza luego, la solicitud del PI en su biblioteca, la que se constituye en biblioteca solicitante (A) y es quien se encarga de realizar el pedido de PI a la biblioteca suministradora (B).

La biblioteca suministradora (B) recibe la petición, la registra y comprueba que realmente dispone del material. En caso afirmativo, lo prepara, registra el préstamo y lo envía a la biblioteca solicitante, en caso negativo le comunica su no disponibilidad.

Cuando el material llega a la biblioteca solicitante (A), se le informa al usuario sobre la recepción y los plazos de préstamo. Una vez finalizada la consulta por parte del usuario, devuelve el material a la biblioteca solicitante (A) quien, por último, gestiona la devolución del material a la biblioteca suministradora (B). Finalmente, en la biblioteca suministradora (B) se registra la devolución del material, se asienta en el sistema de gestión bibliotecaria y finaliza el procedimiento de PI.

\section{Costo del PI}

Los costos que genera el servicio de PI pueden clasificarse en los costos de localización y manejo relacionados a la ubicación y solicitud del documento, en los costos de reproducción cuando la biblioteca suministradora autoriza la copia de algún documento y en los costos de envío que incluyen embalaje, correo, facturación y recepción del documento (Vázquez Valero, 1995: 35). Por lo general, el pago del envío de los documentos es asumido por el usuario que ha solicitado el servicio. Sin embargo, muchas bibliotecas ofrecen el servicio de PI en forma gratuita para sus usuarios y afrontan los costos con sus propios presupuestos.

\section{Cooperación bibliotecaria}

Martín (2004: 4) expresa que "cooperar significa colaborar para obtener un mismo fin y la cooperación bibliotecaria se basa en la premisa de que ninguna biblioteca es autosuficiente y no puede por sí misma satisfacer todas las necesidades de sus usuarios". La cooperación bibliotecaria permite responder al compromiso con la sociedad y contribuir a la mejora educativa. Como refiere Carrión Gutiéz (1993: 424-425) "el éxito de la biblioteca consiste en hacer posible y fácil la comunicación del conocimiento social y la medida de su eficacia es la del aprovechamiento rápido y común de sus fondos". Según Carrato-Mena (2010) el fin de la cooperación es de compartir, rentabilizar recursos y conseguir un mayor nivel de servicios (en cantidad y calidad) y una mayor visibilidad. De tal manera, la cooperación entendida como cualquier actividad que dos o más bibliotecas realizan en forma conjunta, persigue objetivos comunes y pone a disposición el personal y los medios necesarios.

Los elementos básicos para cooperación bibliotecaria de acuerdo a Merlo Vega (1999) son: a) bibliotecas e instituciones que compartan recursos, informaciones y servicios, b) acuerdos que formalicen la colaboración, c) normativa para reglamentar las actividades para el desarrollo del trabajo, d) estructura organizativa en donde todas las bibliotecas se encuentren representadas, e) infraestructura y medios técnicos, f) objetivos, programas y proyectos. 
ABUC contiene los seis elementos necesarios: está formado por 46 bibliotecas miembros, cuenta con un acuerdo-marco, posee una normativa del servicio de PI, se encuentra organizado a través de una estructura organizativa donde todas las bibliotecas se encuentran representadas, dispone de infraestructura y medios técnicos y tiene definido sus objetivos, programas y proyectos.

\section{Resultados}

Se presentan los resultados obtenidos del análisis en base a las dos dimensiones establecidas en los objetivos, en primer lugar, respecto a la gestión del préstamo interbibliotecario, y en segundo lugar, en relación a la percepción de los bibliotecarios y directivos.

\section{A. Respecto a la gestión del préstamo interbibliotecario en general}

\section{a. Tiempo del servicio de PI}

Respecto al tiempo que transcurre entre el pedido y la notificación de la disponibilidad (Tabla1), se relevó que el $88,00 \%$ de las bibliotecas responden dentro de la semana. El $48,00 \%$ responden en el día, el $24,00 \%$ en dos días, el $16,00 \%$ en menos de una semana y el $8,00 \%$ restante demora más de un mes.

Tabla 1: Tiempo que transcurre entre el pedido y notificación de la disponibilidad

\begin{tabular}{lcc}
\hline \multicolumn{1}{c}{ Tiempo } & Frecuencia & $\%$ \\
\hline En el día & 12 & 48,00 \\
En 2 días & 6 & 24,00 \\
Menos de una semana & 4 & 16,00 \\
1 semana & 1 & 4,00 \\
Más de un mes & 2 & 8,00 \\
\hline Total & 25 & 100,00 \\
\hline \multicolumn{3}{c}{ Fuente: Encuesta a encargados }
\end{tabular}

\section{b. Nivel de uso del servicio de PI}

Se tomaron los datos estadísticos del software Cor-pib de los años 2011-2018. Hubo 1.291 de documentos de PI suministrados y 1.156 PI solicitados. En ambos casos, el $97,00 \%$ de los préstamos correspondieron a libros y el 2,30\% a revistas. Existe una diferencia en la cantidad de PI solicitados y suministrados, dado que el software en el ítem suministrados contabiliza la cantidad de documentos, mientras que en solicitados cuenta la cantidad de peticiones. Puede haber solicitudes que se contabilizan como una pero que incluyen varios documentos.

Tres bibliotecas concentraron el 66,46\% de los PI suministrados y cuatro bibliotecas el 70,84\% de los PI solicitados. Por otro lado, se pudo constatar que nueve bibliotecas son las que solicitan más préstamos en comparación con la cantidad que suministran y que catorce bibliotecas suministran más documentos en comparación con la cantidad que solicitan.

Del total de 1.291 PI suministrados por las 26 bibliotecas durante el período de 7 años (2011-2018) se estableció un promedio de 184 PI por año en todo el ABUC y un 
promedio de 7 PI suministrados por biblioteca por año. Luego, del total de $1.156 \mathrm{PI}$ solicitados se determinó un promedio anual de 165 PI y un promedio de 6,3 PI por biblioteca por año.

\section{c. Frecuencia de uso del servicio de PI}

La frecuencia de uso por lo general no tiene una periodicidad marcada (Tabla 2) sino que se establece en base a la demanda de los usuarios (60,00\%). Dos bibliotecas manifiestan tener PI en forma semanal y una en forma quincenal. El resto expresa una frecuencia cada dos meses, tres veces al año, dos veces al año o casi nunca. Las tres bibliotecas que tienen una frecuencia semanal o quincenal coinciden con las bibliotecas que muestran mayor cantidad de PI tanto suministrados como solicitados.

Tabla 2: Frecuencia de uso del PI ABUC

\begin{tabular}{lcc}
\hline Tiempo & Frecuencia & $\%$ \\
\hline Semanalmente & 2 & 8,00 \\
Quincenalmente & 1 & 4,00 \\
Cada dos meses & 1 & 4,00 \\
Tres veces al año & 3 & 12,00 \\
Dos veces al año & 1 & 4,00 \\
Casi nunca & 2 & 8,00 \\
A demanda cuando los usuarios lo solicitan & 15 & 60,00 \\
\hline Total & 25 & 100,00 \\
\hline
\end{tabular}

Fuente: Encuesta a encargados

\section{d. Porcentaje de documentos obtenidos sobre el total de peticiones realizadas}

Según la estimación de los encargados del servicio de PI se obtienen el 78,00\% de documentos sobre el total de peticiones realizadas.

\section{e. Encuesta de satisfacción de usuarios}

Una sola biblioteca realiza encuestas para conocer la percepción del servicio de PI.

\section{f. Sistema de promoción/difusión del servicio de PI}

De las respuestas recogidas se desprende que la mayoría de las bibliotecas, el 72,00\%, realiza acciones de promoción y/o difusión del servicio a través de medios gráficos, carteles, calendarios, de boca en boca, personalmente, correo electrónico, redes sociales, página web, visitas guiadas, presentaciones digitales de los servicios que brinda la biblioteca, cursos o talleres de capacitación o a través de guías para los usuarios.

\section{g. Tipología de usuarios que utilizan el servicio}

En esta pregunta se podía marcar más de una opción. En primer lugar, los alumnos son quienes utilizan mayormente el servicio $(34,32 \%)$, seguido de los docentes $(25,38 \%)$, luego los investigadores $(20,90 \%)$ y finalmente el resto de usuarios. 


\section{h. Tipología de documentos que solicitan}

La mayoría de las bibliotecas solicitan libros (62,16\%), luego revistas $(24,32 \%)$ y en menor proporción archivos electrónicos, CD-ROM o DVD. La respuesta en este ítem coincide con los documentos identificados en el nivel de uso del servicio de PI.

\section{i. Peticiones de préstamos interbibliotecarios $A B U C$ por parte de los usuarios}

Las formas preferidas por los usuarios para solicitar PI (Tabla 3) son: personalmente $(30,90 \%)$, por correo electrónico $(27,27 \%)$, por teléfono $(12,72 \%)$ y por formulario impreso $(10,90 \%)$. Con menor frecuencia se encuentran los pedidos a través de chat, formulario web o correo tradicional.

Tabla 3: Recepción de las solicitudes del PI ABUC por parte de sus usuarios

\begin{tabular}{lcc}
\hline Formas de recepción & Frecuencia & \% \\
\hline Vía correo electrónico & 15 & 27,27 \\
Vía telefónica & 7 & 12,73 \\
Por correo tradicional & 1 & 1,82 \\
Personalmente & 17 & 30,90 \\
Por formulario web & 3 & 5,46 \\
Por formulario impreso & 6 & 10,90 \\
Por chat & 3 & 5,46 \\
No usa PI ABUC & 2 & 3,64 \\
De existir la solicitud se receptaría & 1 & 1,82 \\
personalmente & & \\
\hline Total & 55 & 100,00 \\
\hline
\end{tabular}

Fuente: Encuesta a encargados

j. Recepción de solicitudes de préstamos interbibliotecarios $A B U C$ por parte de las bibliotecas miembros suministradoras

La respuesta a esta pregunta fue de opción múltiple. Las formas preferidas para receptar solicitudes de PI fueron: por correo electrónico $(28,12 \%)$, seguido del software Cor-pib $(23,44 \%)$, por formulario impreso $(18,75 \%)$ y finalmente, por teléfono $(17,19 \%)$.

\section{k. Persona responsable de la gestión del PI}

Los bibliotecarios de servicios al público son quienes generalmente realizan las gestiones de PI (77,78\%). En cinco bibliotecas los responsables son los jefes o coordinadores del área, en otras cinco bibliotecas son los bibliotecarios designados para esa tarea, mientras que en once bibliotecas los responsables son quienes trabajan en atención al público. Se destaca además, que en cuatro bibliotecas son los propios directores los encargados del servicio.

\section{Formas de envío del material solicitado}

Dado que las bibliotecas están distribuidas dentro de la ciudad de Córdoba y en el interior de la provincia, se consideró importante conocer la forma utilizada para el envío de los documentos. Podían contestar más de una opción y de las respuestas recibidas se desprende que el $83,78 \%$ de los casos son los propios usuarios o el personal de la biblioteca solicitante quienes retiran el material. En muy pocos casos se utilizan servicios de correo o de cadetería (Tabla 4). 
Tabla 4: Formas de envío

\begin{tabular}{lcc}
\hline & Frecuencia & \% \\
\hline Es el usuario solicitante quien busca & 16 & 43,24 \\
Es el personal de la biblioteca solicitante & 15 & 40,54 \\
Servicio de cadetería & 3 & 8,12 \\
Ninguna & 1 & 2,70 \\
Servicio postal & 1 & 2,70 \\
Correo interno de la UTN & 1 & 2,70 \\
\hline Total & 37 & 100,00 \\
\hline & \multicolumn{2}{c}{ Fuente: Encuesta a encargados }
\end{tabular}

\section{m. Costo del servicio de PI}

Un factor importante fue conocer el costo asociado a la gestión del PI, dado los magros presupuestos que poseen las bibliotecas. Se indagó acerca de los costos de reproducción y de envío (Vázquez Valero, 1995: 35). En la mayoría de los casos, el $64,00 \%$, la gestión del PI no implica un costo para la biblioteca.

Quienes contestaron afirmativamente la pregunta anterior, respondieron acerca de cómo asume la biblioteca esos costos. De las nueve respuestas, siete expresaron que el costo es asumido por la biblioteca solicitante, uno que el costo lo paga el usuario que solicita el servicio y el otro que el costo es compartido entre la biblioteca y el usuario.

\section{B. Respecto a los bibliotecarios y directivos}

La encuesta a los directivos y encargados de PI tuvo como objetivo identificar a grandes rasgos las fortalezas y debilidades respecto a la logística y a la gestión del servicio, conocer el nivel de satisfacción respecto al software Cor-pib y establecer el nivel de conocimiento respecto al reglamento de PI.

\section{a. Fortalezas y debilidades respecto a la logística y a la gestión del servicio de PI}

Este ítem fue presentado como una pregunta abierta, por eso para la agrupación de las respuestas que aparecieron con mayor frecuencia como fortalezas y como debilidades se utilizó una codificación de letras.

Tabla 5: Fortalezas

\begin{tabular}{|c|c|c|c|}
\hline Código & Descripción & Frecuencia & $\%$ \\
\hline A & Acceso a otras colecciones & 23 & 37,70 \\
\hline C & Cooperación, colaboración & 20 & 32,78 \\
\hline $\mathrm{S}$ & Satisfacción de las necesidades del usuario & 8 & 13,11 \\
\hline I & Mejora la imagen de la biblioteca & 1 & 1,64 \\
\hline $\mathrm{R}$ & Rapidez & 7 & 11,48 \\
\hline Z & Mejora la calidad de servicios & 2 & 3,29 \\
\hline Total & & 61 & 100,00 \\
\hline
\end{tabular}


Tabla 6: Debilidades

\begin{tabular}{|c|c|c|c|}
\hline Código & Descripción & Frecuencia & $\%$ \\
\hline $\mathrm{E}$ & $\begin{array}{l}\text { Problemas con el envío (cadetería, transporte), } \\
\text { costos, usuario que retira, demoras }\end{array}$ & 21 & 47,73 \\
\hline $\begin{array}{c}\mathrm{S} \\
\text { (Cor-pib) }\end{array}$ & $\begin{array}{l}\text { Problemas con el software Cor-pib, poco uso, } \\
\text { poco amigable }\end{array}$ & 9 & 20,45 \\
\hline D & Problemas con la difusión del servicio & 3 & 6,82 \\
\hline C & Problemas con la comunicación & 4 & 9,09 \\
\hline $\mathrm{T}$ & $\begin{array}{l}\text { Problemas con el tiempo (poco tiempo } \\
\text { de préstamo) }\end{array}$ & 3 & 6,82 \\
\hline B & $\begin{array}{l}\text { Problemas con las bibliotecas (no participan } \\
\text { del servicio, falta de predisposición) }\end{array}$ & 4 & 9,09 \\
\hline Total & & 44 & 100,00 \\
\hline
\end{tabular}

Como se puede apreciar en la Tabla 5, las mayores fortalezas son la cooperación y el acceso a otras colecciones, mientras que las mayores debilidades están relacionadas con los problemas de envío y el software Cor-pib (Tabla 6).

\section{b. Nivel de satisfacción respecto al software Cor-pib}

Las respuestas aportadas muestran un alto porcentaje, más del 64,00\% que están satisfechos o muy satisfechos con el software.

\section{c. Conocimiento del instructivo de Cor-pib}

También se consultó si conocían el instructivo que la Comisión Técnica del ABUC elaboró para el uso del software y se observó que un alto porcentaje, el 64,00\% lo conoce.

\section{d. Sugerencias del instructivo de Cor-pib}

Se brindó una pregunta abierta para que los encargados expresaran qué sugerencias aportarían al instructivo. Mientras que varios comentaron que el instructivo es claro, didáctico y que no harían sugerencias, otros mencionaron que es complicado, con mucho texto y que debería contener un vídeo tutorial sobre el uso del sistema.

Si bien muchas de las debilidades mencionadas se refieren al software Cor-pib, los encargados prácticamente no realizaron sugerencias de mejora del instructivo: sólo recomendaron la elaboración de un video y la simplificación de pasos, sin detallar cuáles.

\section{e. Uso del software Cor-pib}

Se consultó acerca si las bibliotecas usan o no el software Cor-pib y los resultados muestran que un alto porcentaje $(72,00 \%)$ contesta afirmativamente. A continuación, se presentan las críticas que los encargados realizaron respecto al software: interface poco amigable y con mucho texto en poco espacio, pantalla compleja y confusa, demasiados pasos para realizar las transacciones. 
A quienes respondieron que no utilizan Cor-pib, se les consultó las razones y expresaron lo siguiente: no tuvieron aún la necesidad, usan papeletas, no disponían de las credenciales de acceso (usuario y contraseña), falta de demanda del servicio.

\section{f. Uso del módulo estadísticas en el software Cor-pib}

El software cuenta con un módulo estadístico, sin embargo, solo nueve bibliotecas lo han utilizado.

\section{g. Registro de estadísticas de PI}

También se consultó si registraban estadísticas de alguna forma distinta a lo establecido por el software Cor-pib y el resultado mostró que más de la mitad (56,00\%) de las bibliotecas miembros registra las estadísticas por otros medios, tales como: planilla Excel, tabla de Word, informes Koha y por carpeta en el correo electrónico.

\section{h. Nivel de conocimiento respecto al reglamento de PI}

Se consultó acerca del conocimiento del reglamento de PI a directivos y a encargados y se verificó que un alto porcentaje contestó en forma afirmativa, el 80,77\% y el 88,00 $\%$ respectivamente.

\section{i. Valoración del PI}

Se indagó respecto al grado de valoración del servicio PI y se solicitó que justificaran la respuesta. En el caso de los encargados, todos manifiestan que el servicio de PI es muy importante, importante o medianamente importante. Pero en los directivos, llama la atención que uno manifiesta que es poco importante y tres expresan no poder valorarlo porque no realizan ellos las gestiones del servicio. Algunas de las respuestas por las cuales valoran positivamente el servicio fueron:

"En la actualidad es de suma importancia formar parte de una red colaborativa de bibliotecas para lograr brindar acceso a la información a la mayor cantidad de documentos posibles. De otra manera, sería muy difícil ya que nuestra biblioteca tiene muy poco espacio y cuenta con muy pocos recursos económicos para la compra del material"

"El servicio tiene un valor único y prácticamente irremplazable por otros servicios"

"Considero que es un servicio sumamente importante, dado que actualmente el paradigma de las bibliotecas es el trabajo en red: compartir, cooperar y colaborar son los pilares de los nuevos servicios."

"Porque es una forma de cooperar y maximiza recursos entre bibliotecas."

"Es un servicio cooperativo que logra que las bibliotecas de nivel universitario puedan compartir y a la vez ser partícipe de la colección de cada una de ellas enriqueciendo a su vez el acervo de cada unidad y logrando satisfacer la demanda de una gran cantidad de usuarios."

"Es altamente relevante para aumentar las colecciones in situ con las de las bibliotecas cooperantes. Esto optimiza recursos, hecho que los organismos de acreditación también promueven porque permite, además, generar vínculos perdurables que promueven nuevos lazos de cooperación." 
"Considero que es uno de los pilares de la asociación. Favorece el intercambio de información por lo que los servicios y propuestas a los usuarios de cada biblioteca son de mejor calidad. Por otro lado, ayuda a conocer y tener una relación fluida con nuestros pares bibliotecólogos."

“Amplía el acceso a la información optimizando recursos. Favorece la interrelación interbibliotecaria y lazos de cooperación. Permite acceder a contenidos de libros ya agotados en el mercado editorial."

"Es una herramienta muy valiosa ya que permite a la biblioteca ofrecer un servicio más completo en cuanto a la cantidad de documentos. Fortalece la comunicación y cooperativismo entre las bibliotecas."

\section{j. Percepción de la satisfacción de los usuarios por parte de PI}

Si bien una sola biblioteca respondió que utiliza encuestas de satisfacción de usuarios, también se preguntó a los directores y a los encargados sobre la percepción que ellos tienen respecto a la satisfacción de los usuarios cuando obtienen el servicio de PI. El $80,77 \%$ de los directores y el $96,00 \%$ de los encargados perciben que los usuarios quedan satisfechos o muy satisfechos con el servicio.

\section{k. Valoración del PI}

$\mathrm{Al}$ indagar sobre la valoración que tienen los directores y los encargados del servicio de PI, se pudo constatar que todos lo perciben como bueno, muy bueno o excelente. Una sola respuesta expresa que no usa el servicio y otra no contesta. Se solicitó que fundamenten la respuesta y algunos de los comentarios fueron:

"La utilidad supera con creces los inconvenientes."

“Para mí el servicio es excelente, tener la posibilidad de conseguir un material que no contamos en nuestra Biblioteca y poder ofrecérselo a nuestros usuarios, es importante, ampliamos nuestro fondo bibliográfico gracias a la colaboración de todas las Bibliotecas participantes."

“Excelente porque su alcance es tal que permite cubrir las necesidades de información no solo con las bibliotecas vecinas o de alrededor sino en bibliotecas del interior."

“Es otra forma más para ampliar nuestra colección."

"Que Instituciones Públicas y Privadas puedan cooperar independientemente de los sistemas de gestión que utilicen es muy valioso."

\section{Conclusiones}

Se puede inferir que el nivel de uso del servicio de PI en los últimos siete años es dispar entre las bibliotecas, dado que existen distintas realidades entre las mismas y que solo tres bibliotecas concentran la mayor cantidad de PI. Existe un alto porcentaje de documentos obtenidos sobre el total de peticiones realizadas y se puede estimar que el tiempo que transcurre entre el pedido de la biblioteca solicitante y la notificación de disponibilidad de la biblioteca suministradora es óptimo dado que responden por lo general dentro de los siete días. Se señala que el usuario recibe la notificación de disponibilidad, pero la obtención del libro no siempre es inmediata y 
dependiendo del lugar donde se ubique la biblioteca suministradora, a veces implica esperar varios días hasta que el documento llega a destino. De todas maneras, de acuerdo con lo investigado las demoras no son excesivas teniendo en cuenta que los usuarios del servicio de PI no solicitan textos de la bibliografía obligatoria, que por lo general las bibliotecas a las que pertenecen ya disponen, sino documentos de consulta e investigación cuya consulta no resulta urgente.

Respecto a las actividades de difusión y/o promoción del PI se constata que la gran mayoría de las bibliotecas realizan difusión a través de distintos medios, pero a excepción de una biblioteca, ninguna utiliza encuestas de satisfacción de usuarios para evaluar el servicio.

Los usuarios que más solicitan el servicio son los alumnos, seguido de los docentes y en menor escala los investigadores. Los libros son más requeridos que las revistas y las peticiones de los usuarios se realizan preferentemente en forma personal, por correo electrónico, por teléfono o por formulario impreso. Mientras que las peticiones por parte de las bibliotecas solicitantes son realizadas en primera instancia por correo electrónico, luego por el software Cor-pib o por formulario impreso y finalmente por teléfono.

Por lo general son los usuarios o el personal de la biblioteca solicitante quienes retiran el material bibliográfico, en menor medida se utilizan los servicios de correo postal o de cadetería.

Respecto a los costos asociados al servicio, por lo general son asumidos por la biblioteca solicitante, pero también en algunos casos el costo es trasladado a los usuarios o son asumidos en forma conjunta entre la biblioteca y el usuario.

La mayor fortaleza detectada del servicio fue la posibilidad de ampliar el acervo bibliográfico de las bibliotecas mediante el acceso a otras colecciones. También se destacó la importancia que en sí misma tiene la cooperación para compartir y maximizar los recursos para lograr una mayor satisfacción del usuario y en consecuencia mejorar la imagen de la biblioteca.

Las mayores debilidades del servicio de préstamos interbibliotecarios estuvieron relacionadas con los problemas relativos al envío y recepción de los documentos. ABUC no dispone de un sistema de cadetería y dado el bajo número de préstamos anuales por biblioteca tampoco se justificaría asumir el costo por el pago de un servicio de traslado de documentos. Esta situación ha llevado a muchas bibliotecas a autorizar a los propios usuarios para retirar el material en la biblioteca suministradora. En otros casos, los propios bibliotecarios y muchas veces los directores de las bibliotecas son quienes transportan los documentos de una biblioteca a otra, especialmente cuando la gestión es realizada con las bibliotecas del interior de la provincia. Las reuniones de la Comisión Técnica de ABUC son las ocasiones propicias para la entrega y devolución del material bibliográfico.

La segunda debilidad planteada fue relativa los problemas con el software de gestión de los préstamos interbibliotecarios Cor-pib. A pesar de que se constató un alto nivel de satisfacción respecto al software, en la consulta sobre su uso surgieron comentarios negativos tales como la interface poco amigable y con mucho texto en escaso espacio, la pantalla compleja y confusa y demasiados pasos para realizar las transacciones.

Finalmente, se enumeran algunas de las sugerencias, ideas o propuestas de mejora del servicio que surgieron de la última pregunta abierta del cuestionario: ofrecer el servicio de préstamos de e-book, concientizar sobre el valor del servicio a las bibliotecas, 
realizar mayor difusión a los usuarios, elaborar un tutorial, eliminar el formulario papel, permitir que los propios usuarios retiren los documentos, modernizar el software Cor-pib para que sea más funcional y práctico, generar alertas desde el software Cor-pib para ambas bibliotecas cuando no se ha completado el procedimiento, mejorar el módulo de estadísticas y capacitar en el uso de Cor-pib. 


\section{Q Referencias Bibliográficas}

》 ABUC. Acuerdo de Bibliotecas Universitarias de Córdoba. 2013. Instructivo Software Préstamo Interbibliotecario (PI). <http://www.abuc.org.ar/portal/ archivos/2013\%20Instructivo-PI.pdf> [Consulta: 20 febrero 2020].

» ABUC. Acuerdo de Bibliotecas Universitarias de Córdoba. 2018a. <http:// www.abuc.org.ar/portal/> [Consulta: 20 febrero 2020].

"ABUC. Acuerdo de Bibliotecas Universitarias de Córdoba. 2018b. Reglamento del Préstamo Interbibliotecario. <http://www.abuc.org.ar/ portal/?sec=2\&pag=30> [Consulta: 20 febrero 2020].

"Carrato-Mena, María Antonia. 2010. Cooperación bibliotecaria para un acceso global al conocimiento. En El Profesional de la Información. Vol. 19, no. 5, 449-454. <http://www.elprofesionaldelainformacion.com/contenidos/2010/ septiembre/o1.pdf> [Consulta: 20 febrero 2020].

»Carrión Gutiéz, Manuel. 1993. Manual de Bibliotecas. Madrid: Fundación Germán Sánchez Ruipérez.

" Magán Wals, José Antonio. 1998. La cooperación bibliotecaria. En Tratado básico de biblioteconomía. Madrid: Editorial Complutense. p. 277-300.

»Martín, Sandra Gisela. 2004. Las bibliotecas universitarias de la provincia de Córdoba: una experiencia de cooperación. Trabajo presentado en el Simposio Electrónico Redes de bibliotecas: oportunidad para la integración. Sociedad Argentina de la Información (SAI), realizado del 6 al 31 de mayo de 2004. <http://eprints.rclis.org/14917/1/2004_Ponencia_ABUC_simposio_SAI_02. pdf> [Consulta: 20 febrero 2020].

"Martín, Sandra Gisela. 2013. Servicios mediados por TICs en la cooperación de bibliotecas universitarias de la provincia de Córdoba. Trabajo presentado en la 16 Encuentro de Bibliotecas Universitarias y la $45^{\circ}$ Reunión Nacional de Bibliotecarios ABGRA, realizado en Buenos Aires del 23 al 25 de abril de 2013. $<$ https://docplayer.es/2514736o-Servicios-mediados-por-ticsen-la-cooperacion-de-bibliotecas-universitarias-de-la-provincia-de-cordoba.html> [Consulta: 20 febrero 2020].

» Martínez de Sousa, José. 1989. Diccionario de bibliología y ciencias afines. Madrid: Fundación Germán Sánchez Ruipérez.

» Merlo Vega, José Antonio. 1999. La cooperación en las bibliotecas universitarias: fundamentos y redes cooperativas. En Boletín de la Asociación Andaluza de Bibliotecarios. No. 54, 33-57. <http://gredos.usal.es/jspui/bitstream/10366/18000/1/DBD_Coop.\%2obtcas.\%20univ..pdf> [Consulta: 20 febrero 2020].

"Vázquez Valero, Manuela. 1995. Suministro de documentos: costes y precios. En Revista Española de Documentación Científica. Vol. 18, no. 1, 33-41. <http:// redc.revistas.csic.es/index.php/redc/article/view/663/738> [Consulta: 20 febrero 2020]. 
